\title{
LDL-C levels in older people: Cholesterol Homeostasis and the Free Radical Theory of Ageing Converge
}

Mark T. Mc Auley ${ }^{1}$ and Kathleen M. Mooney ${ }^{2}$

${ }^{1}$ Faculty of Science and Engineering, Thornton Science Park, University of Chester, CH2 4NU, UK

${ }^{2}$ Faculty of Health and Social Care, Edge Hill University, Ormskirk, Lancashire, L39 4QP, UK 


\section{Abstract}

The cardiovascular disease (CVD) risk factor, low density lipoprotein cholesterol (LDL-C) increases with age, up until the midpoint of life in males and females. However, LDL-C can decrease with age in older men and women. Intriguingly, a recent systematic review also revealed an inverse association between LDL-C levels and cardiovascular mortality in older people; low levels of LDL-C were associated with reduced risk of mortality. Such findings are puzzling and require a biological explanation. In this paper a hypothesis is proposed to explain these observations. We hypothesize that the free radical theory of ageing (FRTA) together with disrupted cholesterol homeostasis can account for these observations. Based on this hypothesis, dysregulated hepatic cholesterol homeostasis in older people is characterised by two distinct metabolic states. The first state accounts for an older person who has elevated plasma LDL-C. This state is underpinned by the FRTA which suggests there is a decrease in cellular antioxidant capacity with age. This deficiency enables hepatic reactive oxidative species (ROS) to induce the total activation of HMG-CoA reductase, the key rate limiting enzyme in cholesterol biosynthesis. An increase in cholesterol synthesis elicits a corresponding rise in LDL-C, due to the downregulation of LDL receptor synthesis, and increased production of very low density lipoprotein cholesterol (VLDL-C). In the second state of dysregulation, ROS also trigger the total activation of HMG-CoA reductase. However, due to an age associated decrease in the activity of cholesterol-esterifying enzyme, acyl CoA: cholesterol acyltransferase, there is restricted conversion of excess free cholesterol (FC) to cholesterol esters. Consequently, the secretion of VLDL-C drops, and there is a corresponding decrease in LDL-C. As intracellular levels of FC accumulate, this state progresses to a pathophysiological condition akin to nonalcoholic fatty liver disease. It is our conjecture this deleterious state has the potential to account for the inverse association between LDL-C level and CVD risk observed in older people. 


\section{Introduction}

Cholesterol is an essential macronutrient which plays an indispensable role in the body. Despite its metabolic utility, plasma cholesterol, particularly elevated levels of low density lipoprotein cholesterol (LDL-C) has a long established relationship with cardiovascular morbidity and mortality (1-4). The standing LDL-C has as a risk factor for cardiovascular health is further emphasized by epidemiological studies which have shown LDL-C increases up until the midpoint of life in males and females $(5,6)$. Conversely, as illustrated in figure 1 it has also been observed in population studies that LDL-C gradually decreases in the latter decades of life $(5,6)$. Why LDL-C increases with age until the midpoint of life and then declines remains unknown. It has been proposed, the rise up until midlife is driven by a combination of intrinsic alterations to whole-body cholesterol metabolism, rather than any single biological change (7). An equally intriguing question centres on a remarkable study of the oldest old (those $\geq 85$ years), where it was observed that both high and low levels of LDL-C had a similar impact on mortality risk (8). Interestingly, this finding arose despite cardiovascular disease (CVD) being the primary cause of mortality in this group. This anomaly was further underscored recently by a somewhat controversial systematic review, which identified two cohorts of older people where CVD mortality was highest in the lowest LDL-C quartile (9).

The studies outlined above raise the possibility of elevated LDL-C diminishing, or even ceasing to be a significant risk factor for CVD mortality in older people. This contradiction is perplexing, as elevated LDL-C is a core component of a paradigm which has been formulated to account for the etiology of atherosclerotic-CVD (10). Traditionally, this model was based on the premise that LDL-C, particularly when elevated accumulated within the innermost layer of the artery wall (11). However, the paradigm has evolved and it is now generally accepted that atherosclerosis is an inflammatory process (12). Atherosclerosis comprises both 
innate and adaptive immunity, and is advanced by lipid accumulation (13). The evolution of this pernicious state involves elevated levels of LDL-C promoting atherosclerosis by binding with proteoglycans in the intima (14). Once LDL is established within the intima, it undergoes several stages of oxidative modification (15). Oxidized lipids and oxidized-LDL trigger an innate immune response eventually culminating in the formation of an atherosclerotic plaque, the defining clinical feature of atherosclerosis $(10,16)$. Thus, elevated LDL-C is firmly established within the widely accepted model which accounts for the onset of atherosclerotic-CVD. However, an explanation which accounts for the findings that LDLC diminishes as a risk factor for CVD in certain older people remains to be fully defined. To address this gap in what is known about cholesterol metabolism, this paper will present a hypothesis which attempts to explain this anomaly. Our idea is based on intracellular cholesterol homeostasis, and its proposed intersection with the free radical theory of ageing. The hypothesis accounts for 1) an increase in LDL-C with age 2) the converse scenario where LDL-C decreases in older people 3) the finding that an inverse relationship exists between cardiovascular mortality and LDL-C in certain older people. 


\section{Premise of hypothesis}

The paradoxical finding that an inverse relationship exists between the risk of cardiovascular mortality and LDL-C in certain older people is thought provoking. Therefore, it is logical to seek a reasonable biological explanation for this conundrum. To address the problem, a worthwhile starting point is the well-established paradigm which accounts for the regulation of plasma LDL-C levels. LDL is the final product of a metabolic cascade which begins with the hepatic flux of very low density lipoproteins (VLDLs) from the liver to the plasma (17). During this process the majority of cholesterol re-enters the liver due to the uptake of VLDL and LDL (17). LDL-C is removed from the circulation by receptor mediated endocytosis (18). The arbiter of this process is the LDL receptor (LDLR), which is strikingly efficient at eliminating circulating LDL-C. Despite the proficiency of LDLRs the uptake of cholesterol is carefully regulated by intracellular cholesterol levels (18). Changes in cellular levels of free cholesterol (FC) provoke two major negative-feedback loops, the first of which acts on 3hydroxy-3-methylglutaryl coenzyme A (HMG-CoA) reductase, which is the key rate limiting enzyme in cholesterol biosynthesis (18). The second negative feedback loop suppresses the synthesis of LDLRs (18). Cholesterol entering the cell from the LDL particle also triggers the cholesterol-esterifying enzyme, acyl CoA: cholesterol acyltransferase (ACAT). Esterification of cholesterol by ACAT2 converts FC to cholesterol esters, which in a hepatic cell are incorporated into VLDL and returned to the plasma (18). By employing these key mechanisms cellular cholesterol levels are subject to tight homeostatic regulation. However, during ageing even the most tightly regulated biological systems are subject to disruption. Due to the complex nature of ageing, unravelling the mechanisms which underpin the impairment of a biological system such as cholesterol homeostasis is challenging. However, it can be argued the free radical theory of ageing (FRTA) is the closest gerontology has come to providing a framework which accounts for the systemic impact of ageing on biological 
systems. The theory was proposed by Harman in 1956, who postulated ageing could be viewed as the result of the accumulation of free radical damage (19). Harman subsequently adapted the theory to suggest free radicals leaking from the mitochondria electron transport chain where the key drivers of ageing (20). This theory has evolved further over the years and is now defined with respect to oxidative stress (21). According to the modern interpretation of the theory, reactive oxidative species (ROS) are usually maintained at low levels within the cell (21). Maintenance of ROS levels is achieved by a subtle balancing act between the rate of production and the rate of scavenging by a plethora of antioxidant systems $(22,23)$. Thus, the contemporary version of the free radical theory suggests ageing results from a decline in the antioxidative capacity of the cell coupled with a concomitant increase in pro-oxidative processes. 


\section{Hypothesis}

It is possible to adopt the contemporary version of the FRTA together with the cholesterol homeostasis paradigm to introduce a hypothesis which accounts for the impact of intrinsic ageing on LDL-C levels in older people. The key elements of this hypothesis are illustrated in figure 2. It is our conjecture, cholesterol biosynthesis in older people is underscored by two different metabolic states. In the first metabolic state hepatic endogenous antioxidant capacity is insufficient to deal with increased ROS levels. The metabolic consequences of this deficiency are that ROS induce the total activation of HMG-CoA reductase, which increases the production of hepatic FC. This elicits a corresponding rise in plasma LDL-C due to the increased secretion of VLDL-C. Such individuals are at risk of developing CVD via the atherosclerosis model outlined previously. In the second scenario which accounts for cholesterol metabolism in very old people, endogenous antioxidant capacity is also overwhelmed by rising intracellular ROS levels. This results in the total activation of HMGCoA reductase; however, the defining feature of this situation is the low activity of ACAT2. The low activity of ACAT2 causes a reduction in the secretion of VLDL-C leading to a decrease in LDL-C production. In our opinion, this metabolic scenario is equally deleterious, because despite a drop in plasma LDL-C levels, intracellular levels of FC accumulate. The build-up of hepatic FC could contribute to the progression of a condition a-kin to nonalcoholic fatty liver disease (NAFLD). NAFLD is associated with an increased risk of developing CVD (24). It is our conjecture that the inverse relationship between the risk of CVD mortality and LDL-C that has been observed in the oldest old can be accounted for by this pernicious metabolic state, which is summarised as follows: Age related diminished hepatic antioxidant capacity $\square$ increased ROS $\square$ total activation of HMG-CoA + drop in 
ACAT2 activity $\square$ reduced secretion of VLDL-C $\square$ a drop in LDL-C production with concomitant increase in hepatic FCØpathophysiological condition akin to NAFLD.

\section{Hypothesis evaluation}

It is cogent to evaluate the evidence both for and against the arguments outlined above. A central challenge to our hypothesis is the FRTA remains controversial. This is emphasized by experimental findings from a broad range of organisms over the last few decades contradicting and supporting the FRTA (25). Therefore, if the claim hepatic antioxidant capacity decreases with age is examined, the literature is to an extent conflicting and inconclusive. For instance, key studies support this idea, such as Semsei et al (1989) who found activities of superoxide dismutase (SOD) and catalase decreased significantly with age in rat liver (26); and Ji (1993) who observed that SOD and glutathione S-transferase activities were significantly decreased in old rodents (27). Juxtapositioned with the idea of diminished antioxidant capacity is the conjecture, ageing is associated with increased ROS production by hepatic mitochondria. This part of the hypothesis is supported by studies, such as that conducted by Sawada et al. (1987) which identified considerable increases in hepatic lipid peroxidation oxidization in the liver of ageing rats (28). Conversely, a significant number of studies have found that endogenous antioxidants and rates of mitochondrial ROS production are low in long-lived animals (29). The proposed biological explanation for this, is that mild mitochondrial oxidative stress render the cell less vulnerable to subsequent oxidative assaults in a process known as mitohormesis (30).

If the idea is examined that ROS are responsible for the total activation of HMG-CoA reductase, evidence for this is sparse but compelling. Pallottini and co-workers suggested ROS have a role to play in the age-related dysregulation of HMG-CoA reductase related to hypercholesterolemia in rats (31). Pallottini et al. (2006) also showed ROS impacted HMG- 
CoA reductase activity in thioacetamide-induced liver injury in rats (32). This group further demonstrated ROS levels influence HMG-CoA reductase dephosphorylation in aged rats (33). Collectively, these results do support the notion that ROS have a significant impact on cholesterol biosynthesis. However, a caveat does exists as ROS may not be the main driver of HMG-CoA reductase activation in females. A study by Trapani et al. (2010) using estropausal rats found that estrogen deficiency in female rats was correlated with higher HMGR activation and did not depend on a rise in ROS, as previously observed in aged-match male rats (34). The authors postulated that lower levels of AMP-activated protein kinase as a consequence of estrogen deficiency was responsible for provoking a decrease in HMGR phosphorylation and subsequent enzyme activation. This is perhaps not surprising as it is known that cholesterol metabolism is intimately connected to hormonal regulation (35). The authors also proposed that several different mechanisms drive dyslipidemia in older men and women (36). However, it can be broadly concluded that ROS have the potential to unregulate HMG-CoA. Explaining why ROS have such a profound effect on this key enzyme is challenging. One interesting explanation from an evolutionary perspective is that sterols played a protective role against oxidative stress in early eukaryote cells (37). Thus, the upregulation of HMGR could be part of an early regulatory mechanism which evolved to protect the cell from increasing levels of oxidation.

The next assertion requiring inspection, is the proposed age-related reduction in ACAT2 activity and its association with both reduced VLDL-C and LDL-C levels. This idea is tentatively supported by Shiomi et al (2000) who examined cholesterol metabolism in homozygous Watanabe heritable hyperlipidemic rabbits (38); an animal model deficient in LDLRs. Despite this deficiency, these rabbits display a considerable decrease in in total plasma cholesterol and LDL-C with age. Shiomi and colleagues identified that a decrease in 
ACAT activity together with a reduction in the secretion rate of VLDL-C, could account for an age related decrease in LDL-C.

According to our hypothesis, a decrease in ACAT activity should also correspond with the accumulation of hepatic FC leading to a condition akin to NAFLD. Notably, it has found been that hepatic FC accumulates in both NAFLD and nonalcoholic steatohepatitis (39). Moreover, Min et al (2012) reported increased cholesterol biosynthesis in NAFLD subjects (40). However, it was also found that cholesterol ester hydrolase increased, ACAT2 levels remained unchanged, and LDLR expression reduced significantly. The fact ACAT2 levels were unchanged is not overly significant for our hypothesis as the mean age of the subjects in this study was $42.5 \pm 7.5$ years and our conjecture is that this enzyme increases significantly in older age. As would be expected in a study of this nature, HMGCR expression was correlated with FC, while LDL-C levels varied directly with HMGCR and inversely with LDLR expression in these patients.

The final aspect of our hypothesis requiring examination is the counter thesis to our standpoint, that both high and low LDL-C levels can present a significant risk to cardiovascular health in older people. Interestingly, despite decades of overwhelming evidence to the contrary recent efforts have been made to establish a case that elevated plasma cholesterol levels do not present a meaningful risk to cardiovascular health $(41,42)$. Thus, an imperative exists to examine this claim, as both a counter argument to our hypothesis and because of its challenge to scientific orthodoxy within the field of cholesterol metabolism. The attraction to this controversial and unconventional idea is somewhat understandable due to some intriguing findings in lipid metabolism over the years. For example, it has been suggested lipoproteins offer a degree of protection from inflammation, possibly by increasing the effectiveness of the immune system via a redistribution of 
cholesterol/ triglycerides to immune cells (43). Another potential avenue which could be used to infer a positive role for high plasma cholesterol levels is the proposal that cholesterol is a circulatory antioxidant. This idea was originally proposed by Smith (1991) who suggested the presence of oxidized oxysterols of human blood represent past interception of blood and tissue oxidants in vivo by cholesterol as a key component of oxygen metabolism (44). This idea also conveniently dovetails with the hypothesis that an early evolutionary role for sterols was in protecting the cell from increasing levels of oxygen. Thus, within such a framework, an altruistic role for cholesterol could be constructed to account for low levels of LDL-C and increased CVD risk. However, given the overwhelming evidence correlating elevated plasma LDL-C levels with atherosclerosis, the suggestion LDL-C is protective appears to be remote.

\section{Investigating the hypothesis}

It would be worthwhile exploring the ideas outlined in this paper in greater depth. However, a significant obstacle to this is that the human liver is subject to a multitude of dynamic processes. Recently, ageing research and lipid metabolism have benefited from adopting the systems biology paradigm (45-48). This framework provides a means of handling the complexities of hepatic cholesterol metabolism and ageing. Key to this approach is the use of mathematical models which enable the integration of disparate experimental data (49-52). This approach overcomes many of the challenges associated with investigating hepatic lipid metabolism and provides an excellent framework for hypothesis exploration.

\section{Consequences of the hypothesis and discussion}

Elevated plasma levels of LDL-C are the gold standard risk factor for CVD. However, in older people LDL-C levels can decrease, and in some instances are inversely associated with 
risk of cardiovascular mortality. It is not known why this happens, however this paradox could mask an underlying disease. Future investigations which examine cholesterol metabolism in the oldest old should give careful consideration to exploring the relationship between LDL-C levels, and conditions such as NAFLD in this population group. Moreover, based on the hypothesis outlined in this paper it is possible extremely low levels of LDL-C could be used as a surrogate marker of hepatic pathology in older people. Further investigations also need to be conducted into the activity of ACAT2 in humans and its potential decline during ageing. In particular, it would be worthwhile investigating if the activity of ACAT2 is also susceptible to ROS. This would provide a mechanism for its dysregulation and help to explain why certain middle aged people have normal/high levels of LDL-C which gradually decrease with age.

\section{References}

[1] W. Castelli, Epidemiology of coronary heart disease: the Framingham study, The American journal of medicine 76 (1984), pp. 4-12.

[2] W.P. Castelli, K. Anderson, P.W. Wilson and D. Levy, Lipids and risk of coronary heart disease The Framingham Study, Annals of epidemiology 2 (1992), pp. 23-28.

[3] W.P. Castelli, R.J. Garrison, P.W. Wilson, R.D. Abbott, S. Kalousdian and W.B. Kannel, Incidence of coronary heart disease and lipoprotein cholesterol levels: the Framingham Study, Jama 256 (1986), pp. 2835-2838.

[4] K.M. Mooney and M.T. Mc Auley, Cardiovascular disease and healthy ageing, Journal of Integrative Cardiology 1 (2015), pp. 76-78.

[5] F.J. Félix-Redondo, M. Grau and D. Fernández-Bergés, Cholesterol and cardiovascular disease in the elderly. Facts and gaps, Aging and disease 4 (2013), p. 154.

[6] M.D. Carroll, D.A. Lacher, P.D. Sorlie, et al., Trends in serum lipids and lipoproteins of adults, 1960-2002, Jama 294 (2005), pp. 1773-1781.

[7] A. Morgan, K.M. Mooney, S.J. Wilkinson, N. Pickles and M.T. Mc Auley, Cholesterol metabolism: A review of how ageing disrupts the biological mechanisms responsible for its regulation, Ageing research reviews 27 (2016), pp. 108-124.

[8] A.W. Weverling-Rijnsburger, I.J. Jonkers, E. van Exel, J. Gussekloo and R.G. Westendorp, Highdensity vs low-density lipoprotein cholesterol as the risk factor for coronary artery disease and stroke in old age, Archives of internal medicine 163 (2003), pp. 1549-1554.

[9] U. Ravnskov, D.M. Diamond, R. Hama, et al., Lack of an association or an inverse association between low-density-lipoprotein cholesterol and mortality in the elderly: a systematic review, BMJ open 6 (2016), p. e010401. 
[10] C. Weber and H. Noels, Atherosclerosis: current pathogenesis and therapeutic options, Nature medicine 17 (2011), pp. 1410-1422.

[11] P. Libby, M. Aikawa and U. Schonbeck, Cholesterol and atherosclerosis, Biochim Biophys Acta 1529 (2000), pp. 299-309.

[12] P. Libby, P.M. Ridker and G.K. Hansson, Progress and challenges in translating the biology of atherosclerosis, Nature 473 (2011), pp. 317-325.

[13] P. Libby, A.H. Lichtman and G.K. Hansson, Immune effector mechanisms implicated in atherosclerosis: from mice to humans, Immunity 38 (2013), pp. 1092-1104.

[14] M.F. Linton, P.G. Yancey, S.S. Davies, W.G.J. Jerome, E.F. Linton and K.C. Vickers, The role of lipids and lipoproteins in atherosclerosis, (2015).

[15] H.S. Kruth, The fate of lipoprotein cholesterol entering the arterial wall, Current opinion in lipidology 8 (1997), pp. 246-252.

[16] I. Tabas, G. García-Cardeña and G.K. Owens, Recent insights into the cellular biology of atherosclerosis, J Cell Biol 209 (2015), pp. 13-22.

[17] S.-y. Morita, Metabolism and modification of apolipoprotein B-containing lipoproteins involved in dyslipidemia and atherosclerosis, Biological and Pharmaceutical Bulletin 39 (2016), pp. 1-24.

[18] J.L. Goldstein and M.S. Brown, The LDL receptor, Arteriosclerosis, thrombosis, and vascular biology 29 (2009), pp. 431-438.

[19] D. Harman, Aging: a theory based on free radical and radiation chemistry, (1955).

[20] D. Harman, The biologic clock: the mitochondria?, Journal of the American Geriatrics Society 20 (1972), pp. 145-147.

[21] D.F. Dai, Y.A. Chiao, D.J. Marcinek, H.H. Szeto and P.S. Rabinovitch, Mitochondrial oxidative stress in aging and healthspan, Longev Healthspan 3 (2014), p. 6.

[22] D. Trachootham, W. Lu, M.A. Ogasawara, N.R.-D. Valle and P. Huang, Redox regulation of cell survival, Antioxidants \& redox signaling 10 (2008), pp. 1343-1374.

[23] J.M. McCord, The evolution of free radicals and oxidative stress, Am J Med 108 (2000), pp. 652-659.

[24] G. Targher and G. Arcaro, Non-alcoholic fatty liver disease and increased risk of cardiovascular disease, Atherosclerosis 191 (2007), pp. 235-240.

[25] J. Vina, C. Borras, K.M. Abdelaziz, R. Garcia-Valles and M.C. Gomez-Cabrera, The free radical theory of aging revisited: the cell signaling disruption theory of aging, Antioxidants \& redox signaling 19 (2013), pp. 779-787.

[26] I. Semsei, G. Rao and A. Richardson, Changes in the expression of superoxide dismutase and catalase as a function of age and dietary restriction, Biochemical and biophysical research communications 164 (1989), pp. 620-625.

[27] L.L. Ji, Antioxidant enzyme response to exercise and aging, Medicine and science in sports and exercise 25 (1993), pp. 225-231.

[28] M. Sawada and J.C. Carlson, Changes in superoxide radical and lipid peroxide formation in the brain, heart and liver during the lifetime of the rat, Mech Ageing Dev 41 (1987), pp. 125137.

[29] R. Perez-Campo, M. Lopez-Torres, S. Cadenas, C. Rojas and G. Barja, The rate of free radical production as a determinant of the rate of aging: evidence from the comparative approach, Journal of Comparative Physiology B 168 (1998), pp. 149-158.

[30] M. Ristow and K. Schmeisser, Mitohormesis: Promoting Health and Lifespan by Increased Levels of Reactive Oxygen Species (ROS), Dose Response 12 (2014), pp. 288-341.

[31] V. Pallottini, C. Martini, A. Pascolini, et al., 3-Hydroxy-3-methylglutaryl coenzyme A reductase deregulation and age-related hypercholesterolemia: a new role for ROS, Mechanisms of ageing and development 126 (2005), pp. 845-851. 
[32] V. Pallottini, C. Martini, A.M. Bassi, P. Romano, G. Nanni and A. Trentalance, Rat HMGCoA reductase activation in thioacetamide-induced liver injury is related to an increased reactive oxygen species content, Journal of hepatology 44 (2006), pp. 368-374.

[33] V. Pallottini, C. Martini, G. Cavallini, et al., Age-related HMG-CoA reductase deregulation depends on ROS-induced p38 activation, Mechanisms of ageing and development 128 (2007), pp. 688-695.

[34] L. Trapani, F. Violo and V. Pallottini, Hypercholesterolemia and 3-hydroxy-3-methylglutaryl coenzyme A reductase regulation in aged female rats, Experimental gerontology 45 (2010), pp. 119-128.

[35] M.T. Mc Auley and K.M. Mooney, Lipid metabolism and hormonal interactions: impact on cardiovascular disease and healthy aging, Expert Review of Endocrinology \& Metabolism 9 (2014), pp. 357-367.

[36] L. Trapani and V. Pallottini, Age-Related Hypercholesterolemia and HMG-CoA Reductase Dysregulation: Sex Does Matter (A Gender Perspective), Current gerontology and geriatrics research (2010), p. 420139.

[37] A.J. Brown and A.M. Galea, Cholesterol as an evolutionary response to living with oxygen, Evolution 64 (2010), pp. 2179-2183.

[38] M. Shiomi, T. Ito, T. Fujioka and Y. Tsujita, Age-associated decrease in plasma cholesterol and changes in cholesterol metabolism in homozygous Watanabe heritable hyperlipidemic rabbits, Metabolism 49 (2000), pp. 552-556.

[39] P. Puri, R.A. Baillie, M.M. Wiest, et al., A lipidomic analysis of nonalcoholic fatty liver disease, Hepatology 46 (2007), pp. 1081-1090.

[40] H.K. Min, A. Kapoor, M. Fuchs, et al., Increased hepatic synthesis and dysregulation of cholesterol metabolism is associated with the severity of nonalcoholic fatty liver disease, Cell Metab 15 (2012), pp. 665-674.

[41] F.A. Kummerow and J.M. Kummerow, Cholesterol is not the culprit: a guide to preventing heart disease (2014).

[42] P. Rosch, Fat and Cholesterol Don't Cause Heart Attacks and Statins Are Not the Solution.

[43] A.M. Barcia and H.W. Harris, Triglyceride-rich lipoproteins as agents of innate immunity, Clinical Infectious Diseases 41 (2005), pp. S498-S503.

[44] L.L. Smith, Another cholesterol hypothesis: cholesterol as antioxidant, Free Radical Biology and Medicine 11 (1991), pp. 47-61.

[45] A. Morgan, K. Mooney, S. Wilkinson, N. Pickles and M. Mc Auley, Investigating cholesterol metabolism and ageing using a systems biology approach, Proceedings of the Nutrition Society (2016), pp. 1-14.

[46] M.T. Mc Auley and K.M. Mooney, Computationally modeling lipid metabolism and aging: a mini-review, Computational and structural biotechnology journal 13 (2015), pp. 38-46.

[47] M.T. Mc Auley, K.M. Mooney, P.J. Angell and S.J. Wilkinson, Mathematical modelling of metabolic regulation in aging, Metabolites 5 (2015), pp. 232-251.

[48] J. Kilner, B.M. Corfe, M.T. McAuley and S.J. Wilkinson, A deterministic oscillatory model of microtubule growth and shrinkage for differential actions of short chain fatty acids, Mol Biosyst 12 (2016), pp. 93-101.

[49] A. Morgan, K.M. Mooney, S.J. Wilkinson, N. Pickles and M.T. Mc Auley, Mathematically modelling the dynamics of cholesterol metabolism and ageing, Biosystems 145 (2016), pp. 19-32.

[50] M.T. Mc Auley, D.J. Wilkinson, J.J. Jones and T.B. Kirkwood, A whole-body mathematical model of cholesterol metabolism and its age-associated dysregulation, BMC systems biology 6 (2012), p. 130.

[51] M.T. Mc Auley, K.M. Mooney and J.E. Salcedo-Sora, Computational modelling folate metabolism and DNA methylation: implications for understanding health and ageing, Briefings in bioinformatics (2016). 
[52] M.T. Mc Auley, C.J. Proctor, B.M. Corfe, C.J. Cuskelly and K.M. Mooney, Nutrition Research and the Impact of Computational Systems Biology, Journal of Computer Science and Systems Biology 6 (2013), pp. 271-285.

Figure 1. An example of how LDL-C rises to the midpoint of life and then declines in later years in the US population (Data taken from reference 5).

Figure 2. Diagrammatic representation of the intersection of the FRTA with Cholesterol homeostasis. Based on our hypothesis a decrease in hepatic antioxidant capacity and a rise in ROS provokes increased cholesterol biosynthesis, which increases LDL-C. Conversely when an increase in ROS is accompanied by a decline in the activity of ACAT2 this results in decreased LDL-C with a concomitant increase in hepatic FC. 